\title{
Tidal disruption events in galactic centers
}

\author{
Resha Parajuli ${ }^{1}$ and Dieter H. Hartmann ${ }^{2}$ \\ ${ }^{1}$ Clemson University \\ email: rparaju@g.clemson.edu \\ ${ }^{2}$ Clemson University \\ email: hdieter@clemson.edu
}

Supermassive black holes $\left(10^{6}-10^{9} \mathrm{M}_{\odot}\right)$ are now known to be present at the centers of most galaxies, but they are also found to have a close correlation with the host galaxy they live in. The masses of the supermasssive black holes (SMBHs) have been rigorously calculated using stellar dynamics (e.g., Gillessen et al. 2009) for the Milky Way, gas dynamics (e.g., Davis et al. 2013) for NGC 4526, water maser emissions, reverberation mapping, etc. In comparison, the mass of the SMBH seems to be tightly correlated with the galactic bulge it resides in. The tight correlation between the mass of the $\mathrm{BH}$ and the velocity dispersion of the stars in the bulge, known as the M- $\sigma$ relation, (Ferrarese \& Merritt, 2000; Tremaine et al. 2002), and the 2:1000 mass of BH - mass of bulge ratio suggests some sort of co-evolution process. A feedback driven coevolution process would suggest that the $\mathrm{BH}$ directly controls galaxy properties via energy and momentum feedback (Kormendy \& Ho, 2013). However, since correlation does not necessarily imply causation, the evolution may be a non-causal process that occurs in tandem, where the $\mathrm{BH}$ and bulge grow independently. In such a process, star formation in the bulge and growth of SMBH occur in separate periods and are self regulating (Cen, 2012).

The process begins with a trigger event such as a major/minor merger that channels a lot of gas to the galaxy's center. Since star formation timescale is much shorter than Salpeter accretion timescale, most of the available gas forms into stars, and the SMBH grows only up to $10 \%$ of its final mass (Cen, 2012). The supernova energy of the once formed stars eventually drives the gas away, and star formation shuts itself off, while the recycled gas is available for the $\mathrm{BH}$ to feed on. After a much longer period of time, ( Gyrs), the SMBH reaches its final value, but continues to grow more steadily. In its quiescent phase, the only material available to the $\mathrm{BH}$ are occasional objects plunging into it. For example, if a star's orbit comes too close to a central BH, it may get deflected into the loss cone, and the BH's gravity may cause the star to tidally shred. This type of stochastic feeding is an important process for the study of the continuous growth of a $\mathrm{BH}$ even after the main supply of fuel has been replenished, and may explain the BH-bulge mass ratio in many galaxies. The intrinsic scatter found in the M- $\sigma$ correlation (Gültekin et al. 2009) may also be the result of a periodic 'co'-evolution.

If a star's point of closest approach to the central BH comes within the tidal radius $\mathrm{R}_{\mathrm{T}} \approx \mathrm{R}_{*}\left(\mathrm{M}_{\mathrm{BH}} / \mathrm{M}_{*}\right)^{1 / 3}$, the star undergoes total disruption. As the star gets torn apart, most of the stellar debris is blown away by the shock front in a super-Eddington outflow. Only a fraction of the material is still bound to the $\mathrm{BH}$ and begins to fall back at a rate proportional to $\mathrm{t}^{-5 / 3}$. The fallback is super-Eddington, and also dictates the luminosity of the flare. The accretion rate decays as a weak power law over time (Lodato \& Rossi, 2011; Cannizzo et al. 1990). The best place to detect a Tidal Disruption Event (TDE) is in low luminosity galaxies as the flares can be as bright as the galaxy, or may even outshine it. The flare resulting from a TDE exhibits a characteristic light curve, lasts from a few days up to a few years, and peaks in the UV/ optical at about $10^{41-43} \mathrm{ergs} / \mathrm{s}$ 
(Gezari et al. 2008; Strubbe \& Quataert, 2009). Apart from its distinctiveness from other transients, only a handful of TDE candidates have been serendipitously discovered so far (Gezari et al. 2008). The rate at which a star may undergo tidal disruption for a typical galaxy is about $10^{-4}$ events/year/galaxy (Magorrian \& Tremaine, 1999). Even though the true rate depends on the mass of the galaxy, we are simply adopting a universal rate for a simple model. With the number density of galaxies in the nearby universe being $10^{-2}$ galaxies $/ \mathrm{Mpc}^{3}$, for $z \sim 1$, we calculated the predicted number of TDE detections to be about 200 events per year.

We are currently working on a simple Monte Carlo model of a sample of galaxies that may experience a TDE at their respective centers. The baseline luminosity of each galaxy is distributed according to the Schechter luminosity function. The galaxies themselves are uniformly distributed in a volume limited sample within $500 \mathrm{Mpc}$. We calculated the flux and apparent magnitudes of these galaxies before and after a tidal disruption event, and found a magnitude difference of up to 5. The best telescopes that can efficiently spot these transients are all sky survey telescopes such as LSST (Large Scale Synoptic Survey), Palomar Transient Factory, Pan-STARRS, etc. For example, LSST has a cadence of only 3 days, and along with its deep and wide field of view, it should be able to detect hundreds of TDEs. Once detected in the optical or UV, it can be followed up with dedicated missions for detailed spectral analysis. Information on the star being disrupted can give further insight into the mass accretion rate and feeding of the central black hole. The next step is to make some progress towards a realistic model of Tidal Disruption Events. TDEs can be used as a probe to study accretion dynamics, the black hole mass function in individual galaxies, tidal disruption rates as a function of black hole mass, and illuminate its significance in the black hole-galaxy co-evolution.

\section{References}

Gillessen, S., Eisenhauer, F., Trippe, S., Alexander, T., Genzel, R., Martins, F., \& Ott, T. 2009, ApJ, 692, 1075

Davis, T., Bureau, M., Cappellari, M., Sarzi, M., \& Blitz, L. 2013, Nature, 494, 328

Ferrarese, L. \& Merritt, D. 2000, ApJ, 539, L9

Tremaine, S., Gebhardt, K., Bender, R., Bower, G., Dressler, A., Faber, S. M., Filippenko, A. V., Green, R., Grillmair, C., Ho, L. C., Kormendy, J., Lauer, T. R., Magorrian, J., Pinkney, J., \& Richstone, D. 2002, ApJ, 574, 740

Kormendy, J. \& Ho, L. C. 2013, ARAA, arXiv:1308,6483

Cen, R. 2012, ApJ, 755, 28

Gültekin, K., Richstone, D. O., Gebhardt, K., Lauer, T. R., Tremaine, S., Aller, M. C., Bender, R., Dressler, A., Faber, S. M., Filippenko, A. V., Green, R., Ho, L. C., Kormendy, J., Magorrian, J., Pinkney, J., \& Siopis, C. 2009, ApJ, 698, 198

Lodato, G. \& Rossi, E. M. 2011, MNRAS 410, 359

Cannizzo, J. K., Lee, H. M., \& Goodman, J. 1990, ApJ 351, 38

Gezari, S., Basa, S., Martin, D. C., Bazin, G., Forster, K., Milliard, B., Halpern, J. P., Friedman, P. G., Morrissey, P., Neff, S. G., Schiminovich, D., Seibert, M., Small, T., \& Wyder, T. K. 2008, ApJ 676, 944

Strubbe, L. E. \& Quataert, E. 2009, MNRAS 400, 2070

Magorrian, J. \& Tremaine, S. 1999, MNRAS 309, 447 